\title{
Evaluating the variation in the projected benefit of community-wide mass treatment for schistosomiasis: Implications for future economic evaluations
}

\author{
Hugo C. Turner ${ }^{1,2,3,4^{*}}$ (D), James E. Truscott ${ }^{1,2}$, Alison A. Bettis ${ }^{1,2}$, Sam H. Farrell ${ }^{1,2}$, Arminder K. Deol ${ }^{1,5}$,
} Jane M. Whitton ${ }^{5}$, Fiona M. Fleming ${ }^{5}$ and Roy M. Anderson ${ }^{1,2}$

\begin{abstract}
Background: The majority of schistosomiasis control programmes focus on targeting school-aged children. Expanding the use of community-wide mass treatment to reach more adults is under consideration. However, it should be noted that this would require a further increase in programmatic resources, international aid, and commitment for the provision of praziquantel. Consequently, it is important to understand (i) where a change of strategy would have the greatest benefit, and (ii) how generalisable the conclusions of field trials and analytical studies based on mathematical models investigating the impact of community-wide mass treatment, are to a broad range of settings.

Methods: In this paper, we employ a previously described deterministic fully age-structured schistosomiasis transmission model and evaluate the benefit of community-wide mass treatment both in terms of controlling morbidity and eliminating transmission for Schistosoma mansoni, across a wide range of epidemiological settings and programmatic scenarios. This included variation in the baseline relative worm pre-control burden in adults, the overall level of transmission in defined settings, choice of effectiveness metric (basing morbidity calculations on prevalence or intensity), the level of school enrolment and treatment compliance.

Results: Community-wide mass treatment was found to be more effective for controlling the transmission of schistosome parasites than using a school-based programme only targeting school-aged children. However, in the context of morbidity control, the potential benefit of switching to community-wide mass treatment was highly variable across the different scenarios analysed. In contrast, for areas where the goal is to eliminate transmission, the projected benefit of community-wide mass treatment was more consistent.

Conclusion: Whether community-wide mass treatment is appropriate will depend on the local epidemiological setting (i.e. the relative pre-control burden in adults and transmission intensity), and whether the goal is morbidity control or eliminating transmission. This has important implications regarding the generalisability of cost-effectiveness analyses of schistosomiasis interventions. Our results indicate that areas with poor school-enrolment/coverage could benefit more from community-wide treatment of praziquantel and should potentially be prioritised for any change in strategy. This work highlights the importance of not over-generalising conclusions and policy in this area, but of basing decisions on high quality epidemiological data and quantitative analyses of the impact of interventions in a range of settings.
\end{abstract}

Keywords: Schistosomiasis, Community-wide treatment, Mass drug administration, MDA, Modelling

\footnotetext{
* Correspondence: hturner@oucru.org

1 London Centre for Neglected Tropical Disease Research, London, UK

2Department of Infectious Disease Epidemiology, School of Public Health,

Faculty of Medicine, St Marys Campus, Imperial College London, Norfolk

Place, London W2 1PG, UK

Full list of author information is available at the end of the article
} 


\section{Background}

Schistosomiasis, also known as snail fever or bilharziasis, is a neglected tropical disease (NTD) caused by parasitic flatworms belonging to the genus Schistosoma. There are two major forms of human schistosomiasis, intestinal and urogenital, which are caused by five main species of blood fluke. Schistosomiasis affects almost 240 million people worldwide, and more than 700 million people are at risk of infection [1]. At present, it is predominantly controlled by school or community-based mass drug administration (MDA) using praziquantel. The majority of schistosomiasis control programmes focus on targeting school-aged children (SAC), via school-based treatment programmes. In some areas, adults are also targeted, ranging from high-risk groups to the entire community [2]. The current World Health Organization (WHO) goals for schistosomiasis are outlined in Table 1.

The global treatment coverage for schistosomiasis is the lowest of all the helminth diseases treated with preventive chemotherapy. Although it has improved recently, schistosomiasis remains classed as red by the annual score card developed by Uniting to Combat NTDs [3]. In 2014 the coverage of at risk SAC and adults was estimated to be 24 and $9 \%$, respectively [4]. This is in spite of a recent increase in the availability of donated praziquantel (Merck $\mathrm{KGaA}$ has now increased its donation of praziquantel to up to 250 million tablets a year, equivalent to 100 million treatments [5]).

There is a growing body of evidence regarding the burden of infection and morbidity in adults, as well as the potential role of these age groups in sustaining transmission [4, 6-11]. This points to a greater need for inclusion of adults in schistosomiasis preventive chemotherapy treatment programmes in some endemic settings. While

Table 1 Overview of the WHO 2013 strategic plan for schistosomiasis [85]

Goals
1. To control morbidity due to schistosomiasis by 2020 (defined as $100 \%$
geographical coverage, $75 \%$ national coverage and < $5 \%$ prevalence
of heavy-intensity infections across all sentinel sites).
2. To eliminate schistosomiasis as a public-health problem by 2025
(defined as a prevalence of heavy-intensity infections $<1 \%$ in all sentinel
sites).
3. To interrupt transmission of schistosomiasis in the Region of the
Americas, the Eastern Mediterranean Region, the European Region,
the South-East Asia Region and the Western Pacific Region, and in
selected countries of the African Region by 2025 (defined as reduction
to zero incidence of infection).
Objectives
1. To scale-up control and elimination activities in all endemic countries
2. To ensure an adequate supply of praziquantel and resources to meet
the demand

expanding the use of community-wide mass treatment to reach more adults is under consideration [12, 13] this would require a further increase in programmatic resources and international commitment. The main bottleneck to schistosomiasis control efforts at present is not the availability of donated praziquantel, but resources and funds for its delivery (especially in remote and hard to reach settings) [14].

Although several field studies have been conducted to investigate the benefit of community-wide mass treatment for schistosomiasis control, findings have been inconsistent [15-18] and some studies find no significant difference when comparing its impact to school-based treatment. Furthermore, Butterworth and colleagues [19] performed studies in Kenya, comparing the long-term impact of different methods of administration of chemotherapy (selective treatment to all infected individuals, selective treatment to individuals with heavy infections, and selective treatment of infected school children). The arm performing selective treatment of all infected individuals showed the greatest relative reductions in infection prevalence and intensity (however, some earlier interventions had been carried out in this area and the pre-treatment intensities were lower than the other arms). The arm providing treatment only to infected schoolchildren also had a marked and prolonged effect (which was comparable to - if not better than - selective treatment of individuals with heavy infections). It was concluded that, in areas of low morbidity, chemotherapy of SAC alone is a satisfactory way of producing a long-term reduction in both infection intensity and morbidity [19].

It is important that we understand the variability of the impact of using community-wide mass treatment across different settings, and in what circumstances it has the greatest benefit (particularly as the relative precontrol burden in adults, has been observed to vary across different settings (Fig. 1) [20]). In this study, we use a mathematical model to evaluate the projected benefit of using annual community-wide mass treatment in terms of both controlling morbidity and eliminating transmission for Schistosoma mansoni (the most prevalent of the schistosome species infecting humans). We investigate how sensitive this projected benefit is to different epidemiological and programmatic assumptions. We also explore how the benefit of switching to community-wide mass treatment is influenced by the chosen effectiveness metric, i.e. what method is used to approximate MDA's impact on morbidity. This is important as there is uncertainty regarding how schistosomiasis models should approximate the impact of treatment on morbidity. The principle goal of this research is to gain an understanding of how generalisable the conclusions of studies investigating the impact and benefit of communitywide mass treatment are across different settings. 


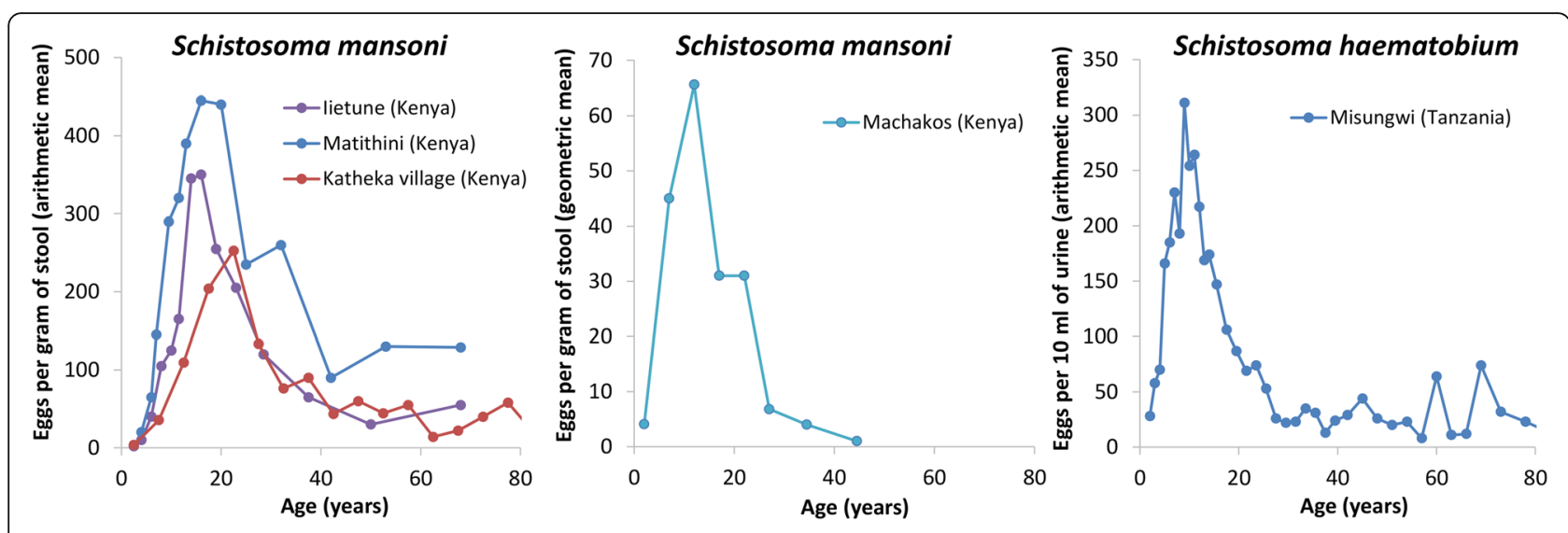

Fig. 1 The observed cross-sectional host-age and mean intensity profiles for Schistosoma mansoni and Schistosoma haematobium infection. The data are from the following sources: lietune (Kenya) [64], Matithini (Kenya) [64], Katheka village (Kenya) [86], Machakos (Kenya) [87], Misungwi (Tanzania) [88]

\section{Methods}

\section{Mathematical model}

In this paper, we employ a previously described deterministic fully age-structured schistosomiasis transmission and MDA treatment model [4, 21], which assumes the parasite is dioecious and monogamous, has densitydependent egg production [22] and a degree of parasite aggregation defined by the negative binomial probability distribution with a fixed $k$ value. The model describes changes in worm burden in order to capture the nonlinear and density dependent processes which influence the effect treatment has on the rate of transmission, such as the worm's mating behaviour and the effect of infection intensity on the female worm's fecundity. The predictions of MDA impact generated by this model are very similar to a more complex individual-based stochastic model [6]. In the model, we assume that the ageintensity profiles (Fig. 2) are generated by age-dependent exposure to the infectious stages in the environment and not acquired immunity [23]. Although the model has full age structure, the outputs are grouped into programmatically meaningful categories such as SAC (5-14 year-olds) and adults ( $\geq 15$ year-olds). The response of the model under treatment has been validated against data (see $[4,6]$ for further details). The model has been adapted to allow for systematic non-compliance (individuals never taking treatment) [24]. Further detail regarding the model and its parameters is provided in Additional file 1.

\section{Model scenarios and sensitivity analysis}

We investigated the impact of an annual school-based treatment programme targeting only SAC, and compared this with an annual community-wide mass treatment programme targeting both SAC and adults. Based on a recent systematic review, we assumed the drug efficacy of a $40 \mathrm{mg}$ per $\mathrm{kg}$ dose of praziquantel was $86.3 \%$ for $S$. mansoni [25]. In line with WHO guidelines, we assumed Pre-SAC were not eligible for treatment [26].

We assumed a $75 \%$ treatment coverage of the targeted age groups and a systematic non-compliance rate of 5\%, i.e. $5 \%$ of the targeted population never take treatment, and the rest of the eligible population take it randomly each round. It should be acknowledged that there are very little data regarding systematic non-compliance rates [27] and we, therefore, varied these assumptions in the sensitivity analysis.

We also considered a scenario where the systematic non-compliance rate was $20 \%$ for the school-based programme, but $5 \%$ when using community-wide mass treatment, simulating a setting where many of the nonenrolled SAC are consistently missed by the school-based

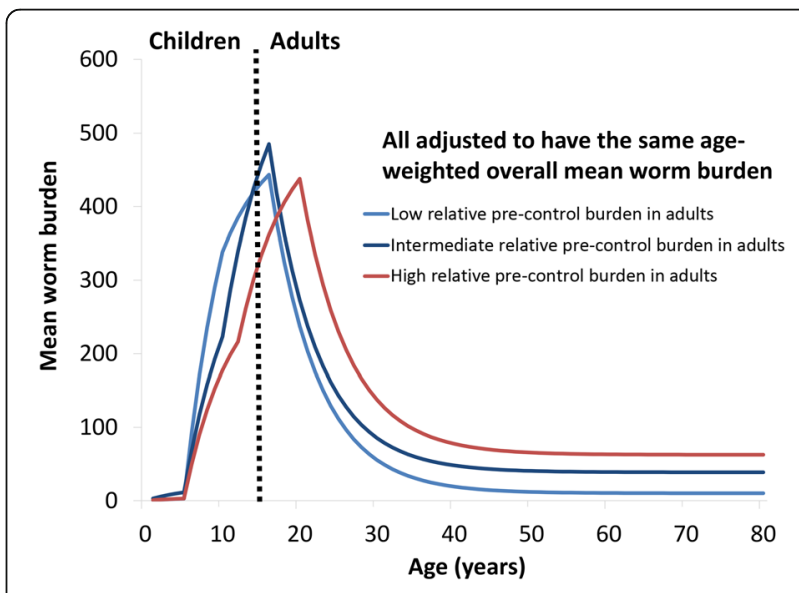

Fig. 2 Model scenarios regarding the age-intensity profiles of infection and the relative pre-control burden in adults. To ensure the results are comparable, the Ro's were adjusted for these different profile scenarios such that they had the same pre-control mean worm burden (i.e. we ensured that we are not comparing the impact of both a different age-profile and a different initial overall burden). Model parameters are shown in Additional file 1: Tables S1 and S2 
programme, and only reached through a communitybased programme.

Based on the available age-intensity profiles (Fig. 1) [6], we defined three scenarios with different levels of relative pre-control infection burdens in adults (low, medium and high, see Fig. 2 and Additional file 1: Tables S1 and S2). These were chosen to represent the observed variation in the relative burden in adults and were based on the fits presented in [6]. It should be noted that these are informed by the limited age-stratified epidemiological data available on infection intensity, and consequently, there may be settings that fall outside of this range. The model was used to simulate two transmission settings: a higher transmission setting with an overall age-weighted mean worm burden of 155, based on model fits to the data [4], and a lower transmission setting with a mean worm burden of 60 . The age-weighted mean egg count per gram of stool (epg) varied between 158 and 166 for the higher transmission setting and 76-78 for the lower transmission setting. The age-weighting accounts for the local demography. To ensure the results for the different scenarios are comparable, the transmission intensity, as represented by the basic reproduction number $\left(R_{0}\right)$, was adjusted such that the different scenarios were based on pre-control mean worm burden, i.e. we ensured that we are not comparing the impact of both a different age-infection profile and a different pre-control burden when comparing the different scenarios. The $R_{0}$ values ranged between 1.30 and 1.32 for the lower transmission setting and 1.63-1.68 for the higher transmission setting.

The sensitivities of the model projections to the assumed life expectancy of the adult worms (4 years instead of 5.71), and the treatment coverage and compliance levels were explored.

\section{Effectiveness metrics}

The model was used to investigate three different effectiveness metrics (modified from [28]) across the chosen time horizon (Fig. 3): (i) the total reduction in the overall worm burden experienced by the population, i.e. number of years lived with a worm (worm years) averted; (ii) the total number of prevalent infection case years averted, i.e. the number of years lived with a prevalent infection prevented; and (iii) the total number of heavy infection case years averted, i.e. the number of years lived with a heavy infection prevented. Heavy infection was defined as having an epg $\geq 400$, an established WHO threshold [2].

We also estimated the number of rounds of preventive chemotherapy required to eliminate transmission - defined as crossing the breakpoint in transmission where infection levels settle to the equilibrium of extinction [29].

\section{Results}

\section{Impact of school-based treatment}

Annual school-based treatment was projected to notably reduce both the worm burden and prevalence of heavy infections in SAC. It also has an indirect effect on the untreated adults due to reductions in transmission (Figs. 4 and 5). However, the overall impact of schoolbased treatment was found to be dependent on the relative pre-control burden in adults (the shape of the age intensity of infection profile) and in many settings a significant burden would remain even after five years of treatment (Figs. 4 and 5). The higher the level of transmission, the greater the significance of this untreated burden in adults (Figs. 4, 5 and Additional file 1: Figure S1).

\section{Benefit of community-wide mass treatment stratified by effectiveness metric}

A community-wide mass treatment strategy was found to increase the effectiveness of preventive chemotherapy against schistosomiasis, though its benefit relative to school-based treatment was found to vary across the different epidemiological scenarios and the employed effectiveness metrics used to quantify the intervention's impact on morbidity (Table 2). The main prediction was that

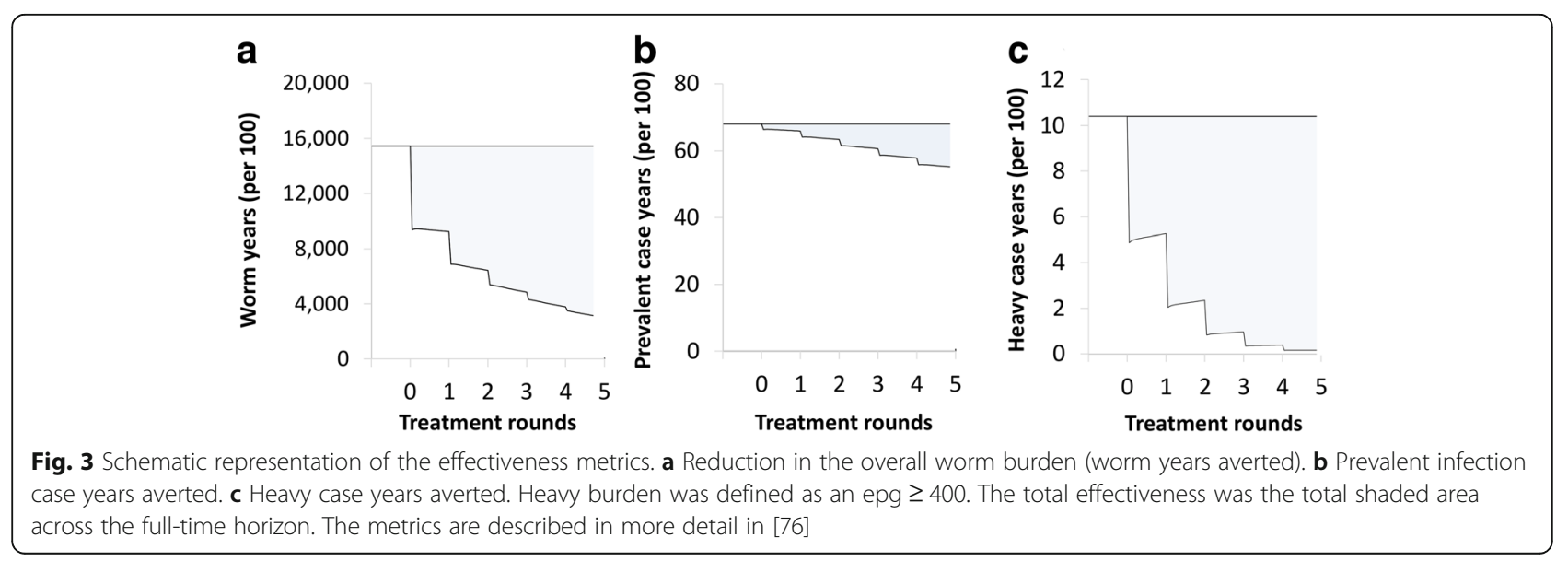




\section{Higher transmission setting $\quad$ Pre-control After 5 years of treatment}

Relative pre-control worm burden in adults

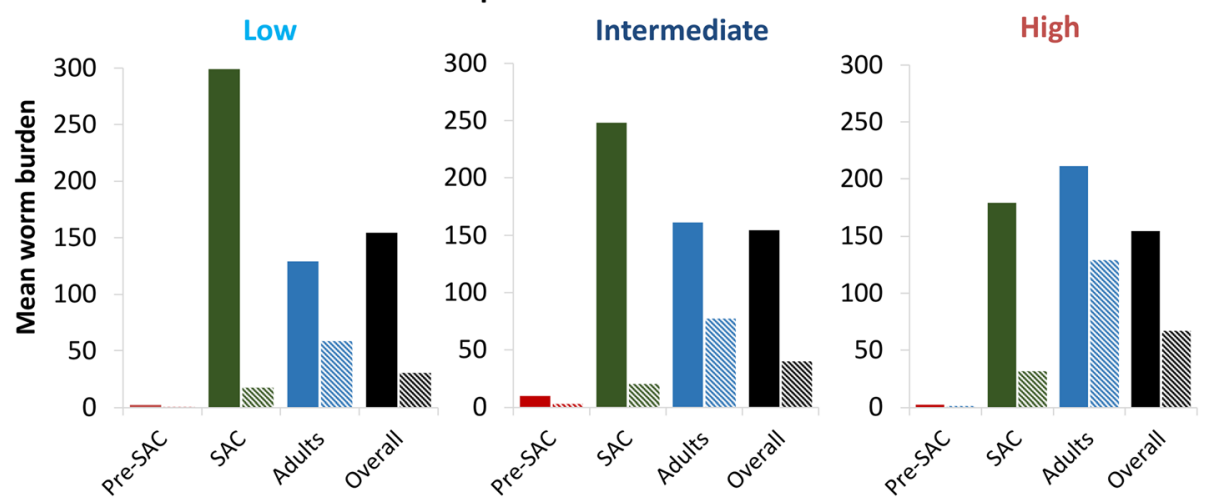

Fig. 4 The impact of annual school-based treatment on the mean worm burden in different age groups. The solid bars represent the pre-control burden and the hashed bars, the burden after 5 years of treatment. The scenarios for the relative pre-control burden in adults are shown in Fig. 2 (note they have the same age-weighted overall mean worm burden). The results assume a treatment coverage of $75 \%$ and $5 \%$ systematic non-compliance. The results pertaining to the lower transmission setting are shown in Additional file 1: Figure S1. Abbreviation: Pre-SAC: 2-4 year-olds, SAC: $5-14$ year-olds and adults: $\geq 15$ year-olds

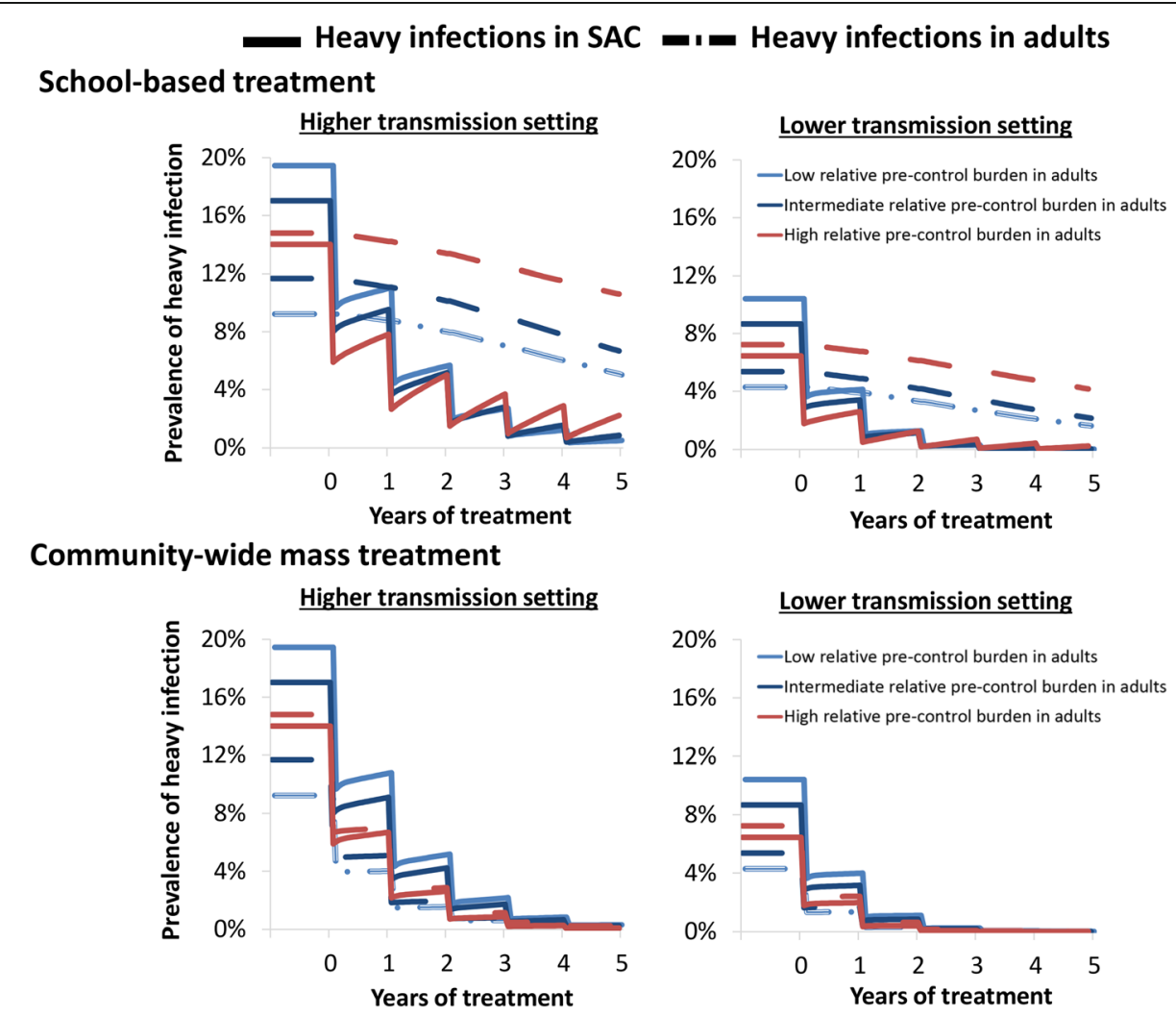

Fig. 5 The impact of annual school-based treatment and community-wide mass treatment on the prevalence of heavy infections in SAC and adults. The solid lines represent the prevalence of heavy infection in SAC and the dashed the prevalence of heavy infection in adults. The different scenarios for the relative pre-control burden in adults are indicated with different coloured lines (see Fig. 2). Heavy infection was defined as having a burden above the WHO threshold ( $\geq 400$ epg) [2]. The results assume a treatment coverage of $75 \%$ and $5 \%$ systematic non-compliance. Abbreviation: SAC: $5-14$ year-olds and adults: $\geq 15$ year-olds 
Table 2 Projected incremental increase in effectiveness when using annual community-wide versus school-based treatment

\begin{tabular}{llll}
\hline Effectiveness metric & Relative pre-control & \multicolumn{2}{l}{ Incremental increase in effectiveness (relative increase in effectiveness) } \\
\cline { 3 - 4 } & Worm burden in adults & Higher transmission setting & Lower transmission setting \\
\hline $\begin{array}{llll}\text { Average number of worm-years } \\
\text { averted per person }\end{array}$ & Low & $306(17 \%)$ & $107(15 \%)$ \\
& Medium & $481(30 \%)$ & $314(59 \%)$ \\
Prevalent case years averted & High & $948(80 \%)$ & $176(70 \%)$ \\
(per 100 individuals) & Medium & $146(83 \%)$ & $223(99 \%)$ \\
& High & $168(124 \%)$ & $305(258 \%)$ \\
Heavy case years averted & Low & $237(303 \%)$ & $8.1(12 \%)$ \\
(per 100 individuals) & Medium & $27.1(23 \%)$ & $11.4(18 \%)$ \\
& High & $44.5(43 \%)$ & $26.7(52 \%)$ \\
\hline
\end{tabular}

The scenarios for the relative pre-control burden in adults are shown in Fig. 2 (note they have the same age-weighted overall mean worm burden). The results assume a treatment coverage of $75 \%$ and $5 \%$ systematic non-compliance. The analysis was conducted with a five-year implementation period and a 15 -year time horizon (i.e. looking at the effect of five years of treatment for 15 years)

scenarios with a higher pre-control burden in adults have a greater relative benefit of using community-wide mass treatment (Table 2). The estimated relative increase in effectiveness was highest when using prevalent case years averted as the effectiveness metric, and was considerably lower in many settings when using the metrics based on infection intensity (either worm years or heavy infection case years averted). For example, when assuming a relatively low pre-control burden in adults, the increase in the number of worm-years averted when using community-wide mass treatment was only 15$17 \%$, in contrast to an increase of $70-83 \%$ for the number prevalent case years averted.

The incremental increase in effectiveness in terms of worm years and heavy infection case years averted was larger in high transmission settings (Table 2). However, the incremental increase in the number of prevalent case years averted was higher in lower transmission settings (Table 2).

In high transmission settings, we found that using community-wide treatment was not only more effective for reducing the overall burden of heavy infections but also more effective for controlling the prevalence of heavy infections specifically in children (Fig. 5).

\section{Sensitivity analysis}

The key results were found to be robust with respect to the sensitivity analyses performed - though the precise estimated benefit of community-wide mass treatment showed some variation (Additional file 1: Tables S3-S6). Of particular note was, the projected benefit of switching to community-wide mass treatment decreased when assuming a lower coverage of adults (Additional file 1: Table S3). In contrast, when assuming a scenario where community-wide treatment would decrease the level of systematic non-compliance in SAC, its benefit increased (Additional file 1: Table S6).

\section{Impact on transmission and projected rounds to elimination} The impact of school-based treatment on the overall level of transmission varied in the different scenarios. However, even when assuming a relatively low precontrol burden in adults and a high coverage of SAC, the reservoir in the untreated adults and SAC still had important implications for the level of ongoing transmission (Fig. 6). In other words, even though these lower intensity infections may not always be sufficient to justify community-wide mass treatment when focussing on morbidity control, they can become significant when trying to break transmission (Fig. 6). These results are mirrored when looking at the number of rounds required to break transmission (Fig. 7) and suggest that when the goal is eliminating transmission, community-wide mass treatment is the best strategy in most settings. However, the results indicate that in high transmission settings it may not be feasible to break transmission with annual preventive chemotherapy alone (Fig. 7 and Additional file 1: Figure S2) [4, 6].

\section{Sensitivity analysis}

When assuming a higher rate of systematic noncompliance (such as 20\%), achieving elimination was projected to be less feasible, requiring more treatment rounds and higher coverage rates (Additional file 1: Figure S2). If a lower mean life expectancy of the adult worms was assumed during the model fitting (4 years instead of 5.71), the projected number of rounds to elimination was generally slightly higher, though the overall findings remained consistent (Additional file 1: Figure S3). 


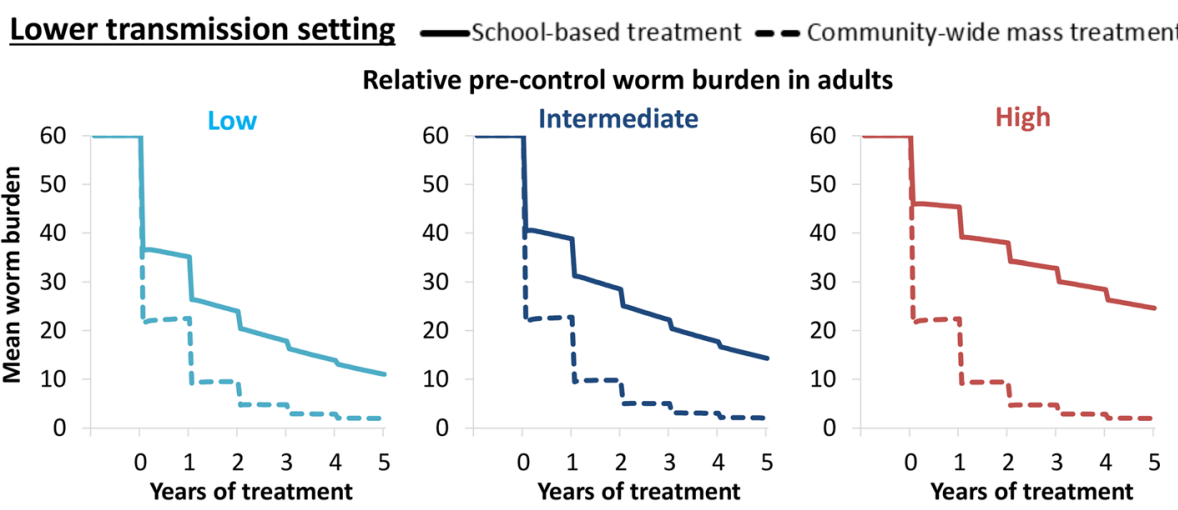

Fig. 6 Comparison of the impact of annual school-based treatment and community-wide mass treatment on the overall mean worm burden. The scenarios for the relative pre-control burden in adults are shown in Fig. 2 (note they have the same age-weighted overall mean worm burden). The results assume a treatment coverage of $75 \%$ and $5 \%$ systematic non-compliance

The results presented in Fig. 7 and Additional file 1: Figures S2-S3 assumed that the rate of systematic noncompliance was the same, for both school-based and community-based treatment programmes. However, it is possible that expanding treatment from schools into the community to reach adults could also increase the number of SAC regularly receiving treatment (as nonenrolled SAC could also be reached more effectively).
In such circumstances, the benefit of expanding treatment into the community for eliminating transmission would be greater (Fig. 8).

\section{Discussion}

The benefit of community-wide mass treatment

Community-wide mass treatment was found to be more effective for controlling the transmission of schistosome

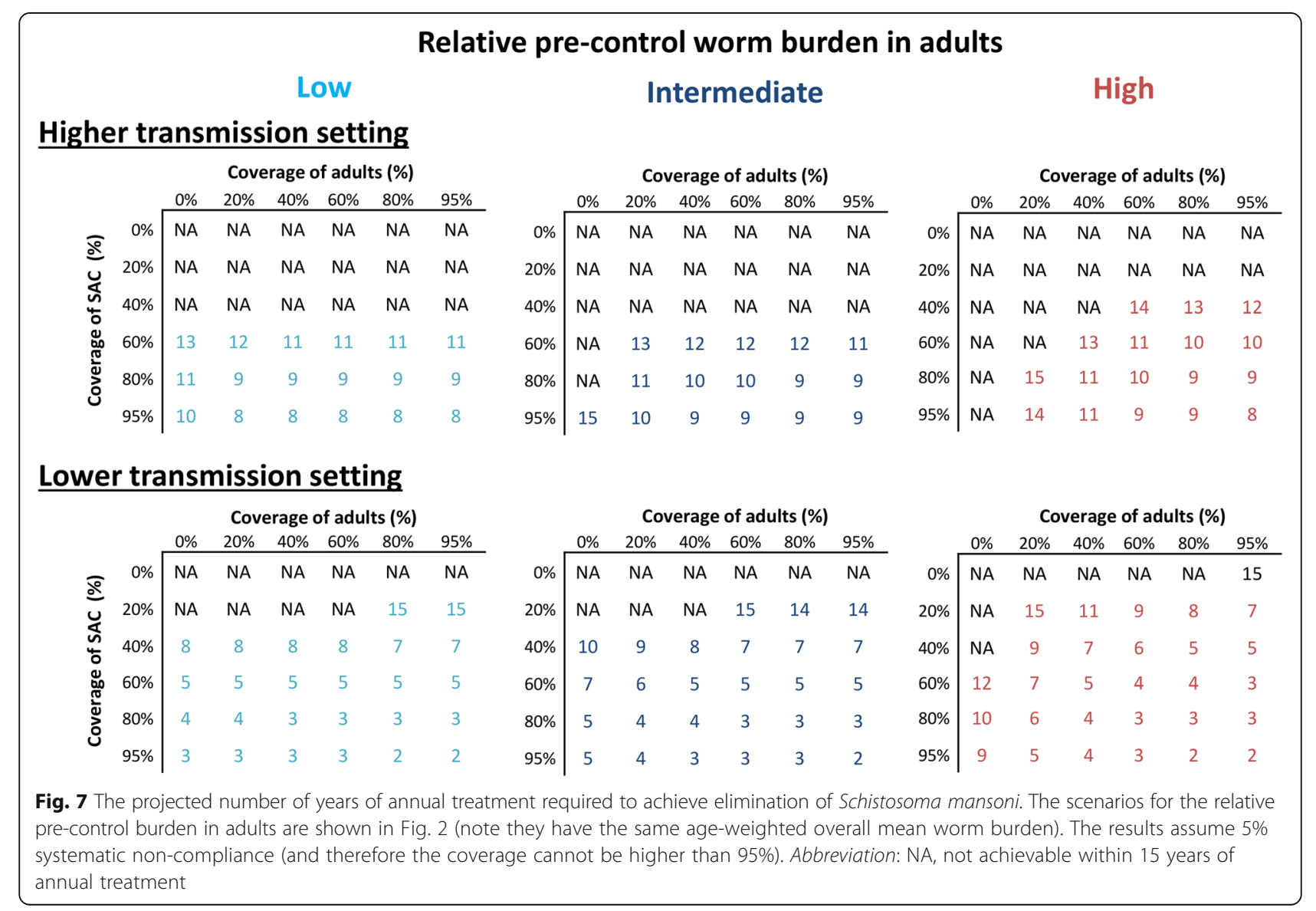




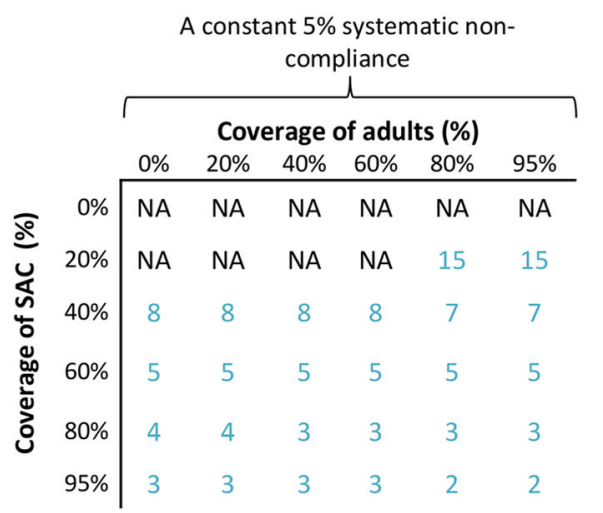

\begin{tabular}{|c|c|c|c|c|c|c|}
\hline \multirow[t]{3}{*}{$\begin{array}{l}20 \% \text { of SA } \\
\text { always mi } \\
\text { school-bas } \\
\text { programm } \\
\text { poor scho } \\
\text { enrolmen }\end{array}$} & $\begin{array}{l}\text { are } \\
\text { sed by } \\
\text { ed } \\
\text { (due }\end{array}$ & $\begin{array}{l}\text { the } \\
\text { to }\end{array}$ & \multicolumn{4}{|c|}{$\begin{array}{l}\text { Systematic non-compliance } \\
\text { reduced to } 5 \% \text { when using } \\
\text { community-wide treatment } \\
\text { (as the unenrolled SAC } \\
\text { reached more effectively) }\end{array}$} \\
\hline & & Cov & erage $c$ & $f$ adu & ts (\%) & \\
\hline & $0 \%$ & $20 \%$ & $40 \%$ & $60 \%$ & $80 \%$ & $95 \%$ \\
\hline \multirow{6}{*}{ 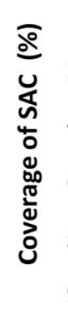 } & NA & NA & NA & NA & NA & NA \\
\hline & NA & NA & NA & NA & 15 & 15 \\
\hline & 10 & 8 & 8 & 8 & 7 & 7 \\
\hline & 7 & 5 & 5 & 5 & 5 & 5 \\
\hline & 6 & 4 & 3 & 3 & 3 & 3 \\
\hline & - & 3 & 3 & 3 & 2 & 2 \\
\hline
\end{tabular}

Fig. 8 Potential impact of annual community-wide mass treatment decreasing the rate of systemic non-compliance in school-aged children. The results pertain to the scenario with a relatively low pre-control worm burden in adults (Fig. 2). When assuming a systematic non-compliance rate of 20\%, it is not possible to get a $95 \%$ coverage of SAC (indicated by a dash in the figure). Abbreviation: NA, not achievable within 15 years of annual treatment

parasites, than using a school-based programme only targeting SAC. However, in the context of morbidity control, the potential benefit of switching to community-wide mass treatment was variable across the different scenarios explored. The benefit was found to be highly dependent on the transmission intensity, the level of school enrolment/ compliance to treatment, and the relative pre-control worm burden in adults. This has important implications regarding the generalisability of any cost-effectiveness analysis of schistosomiasis interventions. The results indicate that in some settings the indirect benefits of a school-based programme on the untreated adults may mean that community-wide mass treatment is unlikely to be more cost-effective in terms of preventing morbidity. This implies that, in settings where the goal is morbidity control, the best strategy may be to scale-up the geographical coverage of the school-based programmes (which is currently low in many settings [30]) and prioritise community-wide mass treatment in settings where the burden in adults and transmission intensity are known to be high, or where school enrolment is poor.

It is important to note that though regular annual community-wide mass treatment may not always be advisable for morbidity control, this does not mean that high-risk adults should not be targeted when possible, particularly pregnant women $[31,32]$ and those in highrisk occupations, such as fishermen. Where resources are too limited to permit expansion to community-wide mass treatment, a potential solution could be to further encourage treatment of the parents of SAC within the school-based programmes or through other existing platforms such as Child Health Days.
Longer term, there is a shifting emphasis towards transmission elimination by WHO. With this aim, our analyses clearly show a benefit of community-wide mass treatment in most situations and it indicates that it would be required in order to succeed within a feasible time frame in most settings - with the exception of low transmission settings where there is little infection in adults. However, the projections also indicate that in high transmission settings it may not be possible to break transmission using annual rounds of preventive chemotherapy alone, and using other strategies such as increasing the treatment frequency, health education, WASH, and snail control, should also be considered $[4,6,12]$. When evaluating the costeffectiveness of incorporating these alternative strategies, it will be important to consider the benefit of preventing hotspots reseeding the infection to other areas and communities.

\section{School-enrolment}

School enrolment rates vary considerably both within and between sub-Saharan African countries. For example, UNICEF reports a number of settings where the net attendance rates are lower than $70 \%$ (with some as low as $21 \%$ ), as well as a large disparity between urban and rural areas [33]. It is also important to consider that children may enrol in primary school but, due to unpaid school fees or seasonal work have to drop out [33]. For example, in Malawi 93\% of children will enrol in primary school; however, only $48 \%$ will complete it [33]. Consequently, in the most marginalised and rural communities within a country very few children may be completing primary school. 
Using a community-wide mass treatment strategy would likely improve the coverage and compliance of non-enrolled SAC - who can be missed when only using a school-based strategy. Analyses indicate that this can have notable implications for the benefit of switching to community-wide mass treatment for both morbidity control and eliminating transmission (Fig. 8 and Additional file 1: Table S6). This further highlights that areas with poor school-enrolment/coverage (or with high school dropout rates) should be prioritised for any shift to communitywide treatment.

\section{Variation in the observed and predicted impact of community-wide mass treatment}

A fundamental reason why studies [15-18] find contrasting results regarding the benefit of community-wide mass treatment is the variation in the relative worm burden harboured by adults across different geographical settings; the higher the pre-control burden in adults, the larger the benefit of switching to community-wide treatment (Fig. 1). A second and often overlooked reason is the way the trial or model is implemented. For example, the age grouping used for the different treatment categories, how the data are categorised, and the time horizon for the analysis, can all influence the estimated strength of the indirect benefit of school-based treatment on the untreated adults. Consequently, these influence the benefit of switching to community-wide mass treatment.

When evaluating different interventions against schistosomiasis, it is vital to account for the shape of the ageintensity profile prior to control. This is illustrated in Fig. 9, which compares the model's projected impact of school-based treatment when (a) the model is fitted to fully age-structured data, which therefore accounts for the true shape of the age-intensity profile, and (b) the model is only fitted to the mean burdens of the SAC and adult age groups, i.e. using the summary statistics from the same dataset for these two age classes. This shows that if the shape of the infection profile is not accounted for, the burden of infection in different age groups and the impact of different interventions can be incorrectly quantified (Fig. 9). Specifically, not accounting for the infection profile's shape can lead the model to underestimate the impact of school-based treatment (Fig. 9). In the example shown in Fig. 9, the more simple fitting method would result in the model overestimating the long-term incremental effectiveness of communitywide mass treatment between 29 and $42 \%$ for the different metrics investigated (Additional file 1: Table S7). This highlights the importance of using fully age-structured models in analyses investigating the impact of targeting different age groups, particularly for cost-effectiveness analyses. The difference between the two fitting methods will vary across different settings and will be dependent on the shape of the infection profile.

We would also like to highlight that it is important to understand in which individuals the burden of heavyintensity infections remains after school-based treatment. For example, Fig. 9 shows that for the modelled setting, the majority of heavy infections remaining are in 20-30 year-olds. If a treatment intervention could be targeted at this remaining high-risk group, expanding to the whole community could be unnecessary for morbidity control in some settings, allowing resources to be redirected to improving coverage and compliance.

\section{Effectiveness metrics and morbidity control}

The benefits of switching to community-wide mass treatment for schistosomiasis morbidity control were found to be very dependent on the choice of the effectiveness metric. i.e. what method has been used to approximate treatments impact on morbidity.

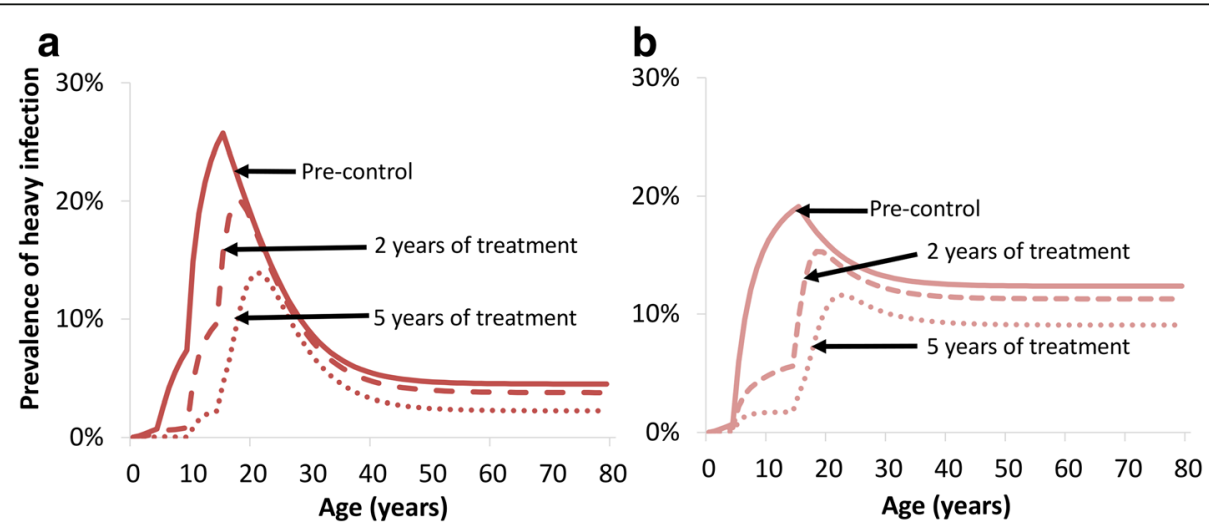

Fig. 9 Modelling the indirect benefit of annual school-based treatment on the prevalence of heavy infection. a The model was fitted to fully age-structured data (and therefore accounts for the true shape of the age-intensity profile) [4]. b The model was only fitted to reproduce the estimated mean pre-control worm burdens in SAC and adults from the same dataset. The data are from the lietune village (Kenya) [64] (Fig. 1). The results assume a treatment coverage of $75 \%$ and no systematic non-compliance 
The Disability-Adjusted Life Year (DALY) burden of schistosomiasis is often calculated by simply applying a disability weight, representing the disability of an 'average' prevalent case of schistosomiasis, to the prevalence of infection. However, it is important to note that the aim of disease burden studies (such as the global burden of disease (GBD) [34]) is to approximate the disease burden at a given point in time. We believe that for schistosomiasis it is misleading to apply this same framework to estimate the morbidity averted over time due to an intervention, i.e. calculating the number of DALYs averted by applying a disability weight to the number of detectable prevalent-case years averted. This is because the morbidity associated with schistosomiasis is complex and often not due merely to the presence or absence of infection [35-50]. Even the early stages of schistosomiasisrelated morbidity (such as diarrhoea, anaemia, and calorie undernutrition), have been found to have a relationship (at least in part) to the individual's intensity of infection [46-51]. This is important since when morbidity is related to the intensity of infection, estimating the impact of treatment on morbidity based solely on reductions in infection prevalence may result in a misleading quantification, particularly regarding the impact of treating different age groups. The key reasons for this are as follows:

(i) Considering only reductions in prevalence assumes that all infections are equally pathogenic and that reducing the intensity of someone's infection but not curing it, has no health benefit. This is particularly significant for this research question as infection intensity tends to decrease in older age groups (Fig. 1). Furthermore, if infections are more pathogenic in children, estimating reductions in morbidity based on reductions in prevalence alone could overestimate the benefit and cost-effectiveness of switching to community-wide treatment. It should be noted that a recent systematic review and meta-analysis concluded that reductions in egg output are significantly correlated with decreased schistosomiasis-related morbidity [52].

(ii)Due to the nonlinear relationship between infection intensity and prevalence, treatment at high-intensity levels can result in a large reduction in average infection intensity but have only a small impact on prevalence (Fig. 10 [29]). Conversely, at lower intensity levels a small impact on infection intensity will lead to a dramatic reduction in prevalence (Fig. 10).

The nonlinear relationship between infection intensity and prevalence (Fig. 10) is why the number of prevalent case years averted was projected to be higher in the lower transmission setting by our model. This would imply that when modelling reductions in morbidity

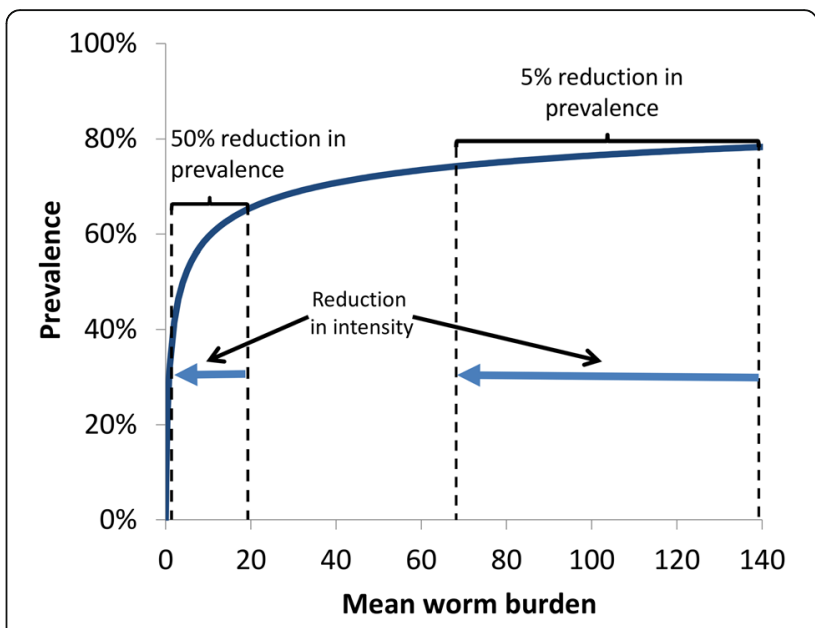

Fig. 10 The relationship between infection intensity and prevalence. The relationship is further described in $[6,29]$

based on reductions in prevalence, the results could find that it is more cost-effective to treat in the lower transmission settings. The other investigated metrics found the opposite relationship and a greater impact in higher transmission settings.

When assessing studies evaluating the burden of schistosomiasis it is also important to consider that late stage morbidity may be permanent (or at least not cured by praziquantel treatment). This means that it is likely that at least some of the morbidity in adults will not be resolved by treatment - as it may be a consequence of the infection burden they had throughout childhood. Previous modelling studies have successfully captured trends in morbidity data using functions which relate morbidity to the accumulated past experience of infection [53-57]. The 2010 GBD study now includes separate calculations for the more advanced forms of schistosomiasis-related morbidity, such as hepatic inflammation, hematemesis, and ascites [58]. However, it is important to note that it does not include advanced urogenital diseases, infertility, or the late effects of growth stunting and cognitive impairment [58].

\section{Burden of light infections}

An important area of uncertainty regarding the benefit of community-wide mass treatment is the potential morbidity associated with active light infections; if their morbidity is high it would increase the benefit of community-wide mass treatment. These will be more frequently identified in field epidemiological studies with the new diagnostic tests (such as the CCA assay [59]) which have a greater sensitivity than egg counts in stool or urine. It is becoming increasingly recognised that the potentially subtle morbidity that results from low-intensity infections may be greater than previously thought and that even low-intensity infections may limit the productivity and reduce the wellbeing of infected adults $[46,52,58,60-62]$. The potential burden of 
these light infections has important implications for the need to shift towards the elimination of transmission [62]. However, although light intensity infections may lead to morbidity $[46,52,58,60,61]$, it is likely that they are more pathogenic in children than adults. Consequently, we believe that to account for the benefit of preventing these active light infections accurately, it is necessary to have a framework which:

(i) Accounts for the differences in the likelihood of morbidity in children versus adults at different levels of infection intensity, i.e. how pathogenic different levels of infection are in different age groups.

(ii) Accounts for which forms of morbidity are permanent (and not cured by treatment).

Without this, any conclusions regarding the benefit of expanding treatment on morbidity would be very dependent on assumptions that are based on limited empirical evidence. Overestimating the relative burden of light infections could overestimate the benefit and cost-effectiveness of switching to community-wide treatment.

It should be stressed that debate continues about whether there is a recognised infection intensity or infection duration threshold below which the risk for disease from Schistosoma infection becomes negligible [60, 61].

The potential morbidity of light infections is also very important regarding the benefit of a paediatric formulation of praziquantel [63]. Previous modelling has suggested that this would be of limited use in bringing about transmission elimination [4]. However, depending on the pathogenicity of these light infections in early childhood, a paediatric formulation of praziquantel could still be highly beneficial for morbidity control.

\section{The most appropriate effectiveness metrics}

The current gaps in knowledge in this area mean that it is difficult to accurately capture the impact of treatment on the morbidity related to schistosomiasis within transmission models. We believe that caution should be employed when interpreting modelling results in this area - which is the reason we did not attempt to estimate DALY averted within this analysis. In our opinion, worm-years (which acts as a metric of the cumulative experience of the population, Fig. 3) and the prevalence of heavy infections are currently the most informative metrics for evaluating the impact of different interventions on schistosomiasis-related morbidity within models. However, as the evidence in this area evolves and more data becomes available, the choice of effectiveness metric should be reassessed/modified, with the concomitant development of frameworks that can accurately estimate the number of DALYs averted.

\section{Limitations in the model projections}

The model predictions reported in this paper were parameterised for S. mansoni. However, given the similar estimates of the life expectancies for other Schistosoma species $[22,64]$ the overall conclusions should be applicable to other species where humans are the dominant host in maintaining transmission.

It should be stressed that the field of schistosome epidemiology suffers greatly from limited information on key parameters such as detailed age-intensity profiles, and the relationship between egg output and worm burden [6]. An important area of uncertainty in the model projections is whether or not acquired immunity against the different schistosome species exists and to what extent it shapes the observed age-intensity profiles [6]. If a species was to generate a strong acquired immunity response, repeated rounds of preventive chemotherapy would reduce the level of herd immunity in an area of endemic infection. Over many rounds of treatment, this would increase reinfection rates (as individuals would not have the same level of past experience of infection and would therefore not gain the same level of immunity), which would act to lessen the long-term impact of preventive chemotherapy $[6,54]$. This relationship could be further complicated if treatment induces acquired immunity [65]. There is also uncertainty regarding the biology of the long-term mating behaviour of the adult worms $[66,67]$ and therefore the most appropriate mating function to use within the models $[6,68]$. Furthermore, it important to note that the model does not account for migration or animal reservoirs. In addition, the model's structure implicitly assumes that hosts contribute infectious material to a single reservoir (that is shared for the entire population) and the degree of parasite aggregation (defined by the negative binomial probability distribution) is assumed to be fixed.

It should also be noted that the scenarios for the relative pre-control burden in adults were informed by the limited age-stratified infection intensity data available and that there may be settings that fall outside this range - including variation of the age at which infection intensity peaks. This highlights the need for more high quality fully cross-sectional data regarding schistosomiasis infection levels in all age classes but especially adults, particularly as the global goals shift to transmission elimination. These data are often lacking due to the programmatic and logistical difficulties of performing the current diagnostic tests in communities.

Currently, the model's prevalence estimates do not account for which infections would be detectable with the available diagnostic tests. When adjusting for this, it is important to account for the fact that the sensitivity of the diagnostic tests, will likely decrease as infection intensity decreases, i.e. the sensitivity of the test is not a constant. 


\section{Programmatic issues and considerations for future economic evaluations}

Currently whether or not adults are targeted within schistosomiasis control programmes is based on the prevalence of infection in SAC [2]. However, in a study in Nigeria [69], the prevalence of infection in SAC, the age group where most monitoring and evaluation activities and data collection is focused, was not a successful indicator of the burden of infection in adults. The pre-control burden in adults will likely be driven by a number of local behavioural and cultural factors, and will, therefore, vary across different countries (and even different regions within countries). This makes it difficult to make a universal SAC infection prevalence threshold for switching to community-wide mass treatment. This further highlights the need for more cost-effective rapid diagnostic tests that allow adults to be sampled more feasibly in a programmatic context [70].

In January of 2012 (as part of the London Declaration on NTDs), Merck KGaA pledged to increase its praziquantel donation from 50 million to 250 million tablets a year for as long as needed [5, 71]. This donation has greatly increased the availability of praziquantel, but it is still less than one-half of the more than 500 million tablets needed annually to treat everyone (children and adults) at risk under the current thresholds for treatment [72]. Potential praziquantel shortages need to be considered when considering the cost of expanding the use of communitywide treatment.

It should be noted that some school-based treatment programmes are financed by the Ministries of Education of endemic countries (and not the Ministries of Health). This needs to be considered when interpreting the conclusions of any cost and cost-effectiveness analysis of switching to community-wide mass treatment - as the same funds may not always be available (which will significantly change the incremental cost of changing strategy).

A further important programmatic consideration for treating continuously across entire communities is the potential risk of drug resistance developing. The current reservoir of untreated worms in adults may be diluting any resistant gene pool in children and therefore expanding treatment could increase the risk of drug resistance. This issue needs careful monitoring with more research to define markers to track via molecular epidemiological studies [73].

One of the most urgent research needs for both schistosomiasis and the soil-transmitted helminths is for detailed costing studies that investigate how the delivery costs of preventive chemotherapy may change when switching to a community from a school-based treatment programme (as well as the potential costs of integrating treatment of adults into other control programmes) [74-76]. In addition, many NTD control programmes are now integrated, and in some settings they may have already established community-delivery platforms which could also be used for schistosomiasis treatment, allowing the treatment of adults at a smaller incremental cost. The potential integration of NTD programmes can have important implications regarding the cost-effectiveness of different strategies $[8,9]$.

Switching to a community-wide mass treatment strategy could be cost-saving in many settings in the long term - due to its capacity to break transmission with fewer treatment rounds (Fig. 7). However, when investigating these potential cost-savings it will be important to consider the risk of human migration resourcing infection; transmission models can be useful in evaluating the risk of this and potential management strategies. It will also be important to consider the implications of potential hybridization of different Schistosoma species, which could increase their geographical range if it changes which snail species are viable intermediate hosts making elimination more challenging [77-79], as well as the potential role of animal reservoirs $[79,80]$.

Further studies are needed to assess the coverage and compliance of different age groups achieved when using different treatment delivery methods [27]. It will also be important to investigate the costs (and achieved coverage) of targeting high-risk adults and how this compares to the costs of targeting the whole community. If a sufficient coverage of high-risk adults could be achieved, it could be a more cost-effective alternative to switching to community-wide mass treatment.

Within this paper, we did not evaluate the benefit of the treating Pre-SAC and this should be explored in future work.

It is also important to recognise that urogenital schistosomiasis may have a role in human immunodeficiency virus (HIV) and human papillomavirus (HPV) infections [81-83] and could potentially make co-infected individuals more infectious [82, 84]. This suggests that community-wide treatment for schistosomiasis could have benefits on other diseases - which are not captured in this study. The potential impact of schistosomiasis on other diseases should be researched further and considered in policy decisions.

\section{Conclusions}

Community-wide mass treatment was found to be more effective for controlling schistosome infection than using a school-based programme only targeting SAC. However, its relative benefit was highly variable across the different scenarios explored. For example, the incremental impact of community-wide mass treatment relative to school-based treatment was very dependent on the local epidemiological setting and the method used to approximate the impact of treatment on morbidity, i.e. was the effectiveness metric based on reductions in infection prevalence or reductions 
in infection intensity. This has important implications regarding the generalisability of cost-effectiveness analyses of schistosomiasis interventions. Due to our limited understanding of the causal link between schistosomiasis morbidity and infection, we believe that caution should be employed when interpreting modelling results regarding the amount of schistosomiasis morbidity averted by different treatment strategies. In particular, our results highlight that basing the effectiveness/disease metric solely on reduced infection prevalence may produce misleading conclusions and that this area needs further research. It is important to highlight that although switching to regular annual community-wide mass treatment may not always be advisable, this does not mean that high-risk adults should not be targeted when possible. For areas where the goal is to eliminate transmission, the projected benefit of community-wide mass treatment was more consistent. Ultimately, whether community-wide mass treatment is appropriate will depend on the epidemiological and programmatic setting, i.e. the relative pre-control burden in adults, school enrolment and transmission intensity, and whether the goal is morbidity control or eliminating transmission. This work highlights the importance of not over-generalising conclusions and policy in this area, but of basing decisions on high-quality epidemiological data and quantitative analyses of the impact of interventions in a range of settings.

\section{Additional file}

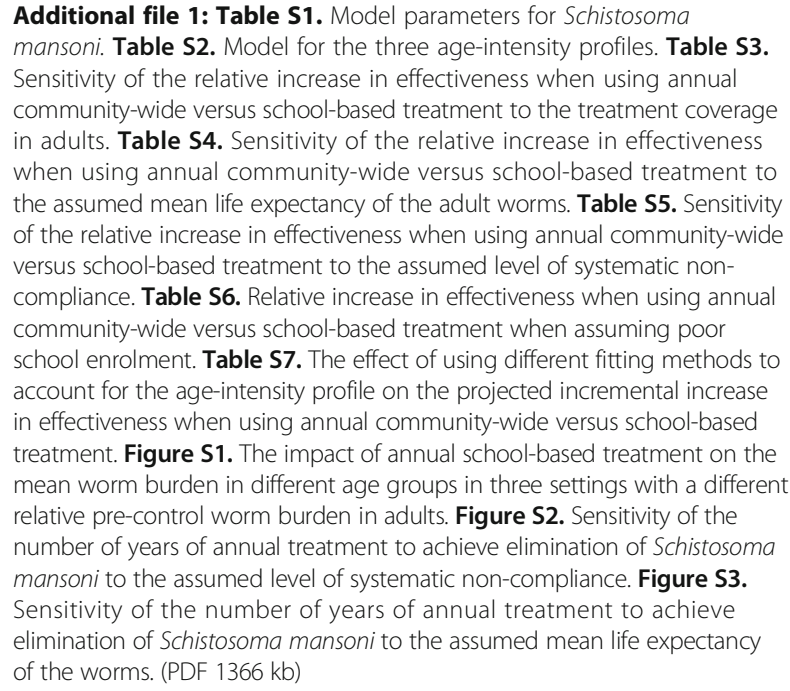
mansoni. Table S2. Model for the three age-intensity profiles. Table S3. Sensitivity of the relative increase in effectiveness when using annual community-wide versus school-based treatment to the treatment coverage in adults. Table S4. Sensitivity of the relative increase in effectiveness when using annual community-wide versus school-based treatment to the assumed mean life expectancy of the adult worms. Table S5. Sensitivity of the relative increase in effectiveness when using annual community-wide versus school-based treatment to the assumed level of systematic noncompliance. Table S6. Relative increase in effectiveness when using annual community-wide versus school-based treatment when assuming poor school enrolment. Table S7. The effect of using different fitting methods to account for the age-intensity profile on the projected incremental increase in effectiveness when using annual community-wide versus school-based treatment. Figure S1. The impact of annual school-based treatment on the mean worm burden in different age groups in three settings with a different relative pre-control worm burden in adults. Figure $\mathbf{S 2}$. Sensitivity of the number of years of annual treatment to achieve elimination of Schistosoma mansoni to the assumed level of systematic non-compliance. Figure S3. Sensitivity of the number of years of annual treatment to achieve elimination of Schistosoma mansoni to the assumed mean life expectancy of the worms. (PDF $1366 \mathrm{~kb}$ )

\section{Abbreviations}

DALY: Disability-adjusted life year; Epg: Eggs per gram of stool; GBD: Global burden of disease; HIV: Human immunodeficiency virus; HPV: Human papillomavirus; MDA: Mass drug administration; NTD: Neglected tropical disease; Ro: Basic reproduction number; SAC: School-aged children; WHO: World Health Organization

\section{Acknowledgements}

We thank Dr Charles King for providing access to the "Katheka village" data in Fig. 1. JET, AKD and RMA gratefully acknowledge funding from The Children's Investment Fund Foundation (UK) ("CIFF"). The views, opinions, assumptions or any other information set out in this paper are solely those of the authors and should not be attributed to CIFF or any person connected with CIFF.

\section{Funding}

HCT and AAB were supported by London Centre for Neglected Tropical Disease Research (funded by GSK). HCT is supported by the Wellcome Trust (089276/B/09/7). The funders had no role in study design, data collection and analysis, decision to publish, or preparation of the manuscript.

\section{Availability of data and materials}

The datasets supporting the conclusions of this article are included within the article and its additional files. The data presented in Fig. 1 are available from the referenced papers (with the exception of the data from "Katheka village", which is available upon request from Dr Charles King).

\section{Authors' contributions}

$\mathrm{HCT}$ conducted the analysis and drafted the first version of the manuscript. JET coded the model and performed the parameter estimation. AAB assisted in drafting the manuscript. JET, AAB, SHF, AD, JWM, FMF and RMA contributed to the design of the study and writing of the paper. All authors read and approved the final manuscript.

\section{Competing interests}

Roy M. Anderson is a Non-Executive Director of GlaxoSmithKline (GSK). GlaxoSmithKline played no role in study design, data collection and analysis, decision to publish, or preparation of the manuscript. The other authors have no competing interests.

\section{Consent for publication}

Not applicable.

Ethics approval and consent to participate

Not applicable.

\section{Publisher's Note}

Springer Nature remains neutral with regard to jurisdictional claims in published maps and institutional affiliations.

\section{Author details}

${ }^{1}$ London Centre for Neglected Tropical Disease Research, London, UK. 2Department of Infectious Disease Epidemiology, School of Public Health, Faculty of Medicine, St Marys Campus, Imperial College London, Norfolk Place, London W2 1PG, UK. ${ }^{3}$ Oxford University Clinical Research Unit, Wellcome Trust Major Overseas Programme, Ho Chi Minh City, Vietnam. ${ }^{4}$ Centre for Tropical Medicine and Global Health, Nuffield Department of Medicine, University of Oxford, Oxford, UK. ${ }^{5}$ Schistosomiasis Control Initiative, Department of Infectious Disease Epidemiology, School of Public Health, Faculty of Medicine (St. Mary's Campus), Imperial College London, Norfolk Place, London W2 1PG, UK.

Received: 20 December 2016 Accepted: 18 April 2017 Published online: 28 April 2017

\section{References}

1. World Health Organization. Schistosomiasis: A major public health problem. http://www.who.int/schistosomiasis/en/. Accessed 20 Feb 2017.

2. World Health Organization. Prevention and control of schistosomiasis and soil-transmitted helminthiasis. World Health Organ Tech Rep Ser. 2002;912:57.

3. Uniting to Combat NTDs: The Fourth Report: Reaching the unreached. 2016 http://unitingtocombatntds.org/report/fourth-report-reaching-unreached. Accessed 20 Feb 2017

4. Anderson RM, Turner HC, Farrell SH, Yang J, Truscott JE. What is required in terms of mass drug administration to interrupt the transmission of schistosome parasites in regions of endemic infection? Parasit Vectors. 2015;8(1):1-11.

5. Global Schistosomiasis Alliance strives for elimination of the worm disease schistosomiasis. http://www.merck.de/company.merck.de/de/images/CRNews 2015_08_07_GSA_tcm1613_140462.pdf?Version=. Accessed 20 Feb 2017. 
6. Anderson RM, Turner HC, Farrell SH, Truscott JE. Studies of the transmission dynamics, mathematical model development, and the control of schistosome parasites by mass drug administration in human communities. Adv Parasitol. 2016:94:199-246.

7. Njenga SM, Mwandawiro CS, Muniu E, Mwanje MT, Haji FM, Bockarie MJ. Adult population as potential reservoir of NTD infections in rural villages of Kwale district. Coastal Kenya. 2011:4:175.

8. Lo NC, Bogoch II, Blackburn BG, Raso G, N'Goran EK, Coulibaly JT, et al. Comparison of community-wide, integrated mass drug administration strategies for schistosomiasis and soil-transmitted helminthiasis: a costeffectiveness modelling study. Lancet Global Health. 2015;3(10):e629-38.

9. Lo NC, Lai Y-S, Karagiannis-Voules D-A, Bogoch II, Coulibaly JT, Bendavid E, et al. Assessment of global guidelines for preventive chemotherapy against schistosomiasis and soil-transmitted helminthiasis: a cost-effectiveness modelling study. Lancet Inf Dis. 2016;16(9):1065-75.

10. Gurarie D, Yoon N, Li E, Ndeffo-Mbah M, Durham D, Phillips AE, et al. Modelling control of Schistosoma haematobium infection: predictions of the long-term impact of mass drug administration in Africa. Parasit Vectors. 2015;8(1):529.

11. Wang X, Gurarie D, Mungai PL, Muchiri EM, Kitron U, King CH. Projecting the long-term impact of school- or community-based mass-treatment interventions for control of Schistosoma infection. PLoS Negl Trop Dis. 2012;6(11):e1903.

12. World Health Organization. Meeting of the International task force for disease eradication, April 2012. Wkly Epidemiol Rec. 2012;87(33):305-9.

13. Lo NC, Addiss DG, Hotez PJ, King CH, Stothard JR, Evans DS, et al. A call to strengthen the global strategy against schistosomiasis and soil-transmitted helminthiasis: the time is now. Lancet Inf Dis. 2017;17(2):e64-9.

14. Uniting to Combat NTDs. From promises to progress: The first anniversary report on the London Declaration on NTDs. http://unitingtocombatntds. org/resource/promises-progress-first-report-london-declaration-ntds. Accessed 20 Feb 2017.

15. Zhang Y, Koukounari A, Kabatereine N, Fleming F, Kazibwe F, Tukahebwa E, et al. Parasitological impact of 2-year preventive chemotherapy on schistosomiasis and soil-transmitted helminthiasis in Uganda. BMC Med. 2007;5(1):1-11.

16. Toure S, Zhang Y, Bosque-Oliva E, Ky C, Ouedraogo A, Koukounari A, et al. Two-year impact of single praziquantel treatment on infection in the national control programme on schistosomiasis in Burkina Faso. Bull World Health Organ. 2008:86(10):780-7.

17. Onkanga IO, Mwinzi PNM, Muchiri G, Andiego K, Omedo M, Karanja DMS, et al. Impact of two rounds of praziquantel mass drug administration on Schistosoma mansoni infection prevalence and intensity: a comparison between community wide treatment and school based treatment in western Kenya. Int J Parasitol. 2016;46(7):439-45.

18. Massa K, Magnussen P, Sheshe A, Ntakamulenga R, Ndawi B, Olsen A. The effect of the community-directed treatment approach versus the schoolbased treatment approach on the prevalence and intensity of schistosomiasis and soil-transmitted helminthiasis among schoolchildren in Tanzania. Trans R Soc Trop Med Hyg. 2009;103(1):31-7.

19. Butterworth AE, Sturrock RF, Ouma JH, Mbugua GG, Fulford AJ, Kariuki HC, Koech D. Comparison of different chemotherapy strategies against Schistosoma mansoni in Machakos District, Kenya: effects on human infection and morbidity. Parasitology. 1991;103(Pt 3):339-55.

20. Schur N, Utzinger J, Vounatsou P. Modelling age-heterogeneous Schistosoma haematobium and S. mansoni survey data via alignment factors. Parasit Vectors. 2011:4:142.

21. Truscott JE, Gurarie D, Alsallaq R, Toor J, Yoon N, Farrell SH, Turner HC, Phillips AE, Aurelio HO, Ferro J, King $\mathrm{CH}$, Anderson RM. A comparison of two mathematical models of the impact of mass drug administration on the transmission and control of schistosomiasis. Epidemics. 2017;18: 29-37.

22. Anderson RM, Medley GF. Community control of helminth infections of man by mass and selective chemotherapy. Parasitology. 1985;90:629-60.

23. Warren KS. Regulation of the prevalence and intensity of schistosomiasis in man: Immunology or ecology? J Inf Dis. 1973;127(5):595-609.

24. Farrell SH, Truscott JE, Anderson RM. The importance of patient compliance in repeated rounds of mass drug administration (MDA) for the elimination of intestinal helminth transmission. Parasit Vectors. 2017. In press

25. Zwang J, Olliaro PL. Clinical efficacy and tolerability of praziquantel for intestinal and urinary schistosomiasis? A meta-analysis of comparative and non-comparative clinical trials. PLoS Negl Trop Dis. 2014;8(11): e3286.
26. World Health Organization. Preventive chemotherapy in human helminthiasis: coordinated use of anthelminthic drugs in control interventions: a manual for health professionals and programme managers. Geneva: WHO; 2006.

27. Shuford KV, Turner HC, Anderson RM. Compliance with anthelmintic treatment in the neglected tropical diseases control programmes: a systematic review. Parasit Vectors. 2016;9(1):29.

28. Medley GF, Guyatt HL, Bundy DA. A quantitative framework for evaluating the effect of community treatment on the morbidity due to ascariasis. Parasitology. 1993;106(Pt 2):211-21.

29. Anderson RM, May RM. Infectious diseases of humans: dynamics and control. Oxford: Oxford Science; 1991.

30. World Health Organization, PCT databank: Schistosomiasis. http://www.who.int/ neglected_diseases/preventive_chemotherapy/sch/en/. Accessed 20 Feb 2017.

31. Friedman JF, Mital P, Kanzaria HK, Olds GR, Kurtis JD. Schistosomiasis and pregnancy. Trends Parasitol. 2007;23(4):159-64.

32. Olds GR. Administration of praziquantel to pregnant and lactating women. Acta Trop. 2003;86(2-3):185-95.

33. Unicef. Education is vital to meeting the Sustainable Development Goals. http://data.unicef.org/education/overview.html. Accessed 20 Feb 2017.

34. Murray CJL, Vos T, Lozano R, Naghavi M, Flaxman AD, Michaud C, et al. Disability-adjusted life years (DALYs) for 291 diseases and injuries in 21 regions, 1990-2010: a systematic analysis for the Global Burden of Disease Study 2010. Lancet. 2012;380(9859):2197-223.

35. Jordan P, Webbe G. Schistosomiasis: epidemiology, treatment, and control. London: William Heinemann Medical Books Ltd; 1982.

36. Arap Siongok TK, Mahmoud AA, Ouma JH, Warren KS, Muller AS, Handa AK, Houser HB. Morbidity in schistosomiasis mansoni in relation to intensity of infection: study of a community in Machakos. Kenya Am J Trop Med Hyg. 1976;25(2):273-84.

37. Lehman Jr JS, Mott KE, Morrow Jr RH, Muniz TM, Boyer MH. The intensity and effects of infection with Schistosoma mansoni in a rural community in northeast Brazil. Am J Trop Med Hyg. 1976;25(2):285-94.

38. Cheever AW, Kamel IA, Elwi AM, Mosimann JE, Danner R. Schistosoma mansoni and S. haematobium infections in Egypt. II. Quantitative parasitological findings at necropsy. Am J Trop Med Hyg. 1977;26(4):702-16.

39. De Cock KM. Hepatosplenic schistosomiasis: a clinical review. Gut. 1986;27(6):734-45

40. Nash TE, Cheever AW, Ottesen EA, Cook JA. Schistosome infections in humans: perspectives and recent findings. NIH conference. Ann Intern Med. 1982;97(5):740-54

41. Warren KS. The pathogenesis of hepatosplenic schistosomiasis: from man to monkey to mouse to molecule. Prog Liver Dis. 1979;6:439-55.

42. Honey RM, Gelfand M. The urological aspects of bilharziasis in Rhodesia. Cent Afr J Med. 1960;6:109-11.

43. Most H, Kane CA, Lavietes PH, Schroeder EF, Behm A, Blum L, et al. Schistosomiasis japonica in American military personnel; clinical studies of 600 cases during the first year after infection. Am J Trop Med Hyg. 1950;30(2):239-99.

44. Sulit YS, Domingo EO, Dalmacio-Cruz AE, Deperalta DS, Imperial ES. Parasitic cirrhosis among Filipinos. J Philipp Med Assoc. 1964;40(Suppl):1021-38.

45. Ostrea Jr EM, Marcelo Jr FB. Relationship between pulmonary schistosomiasis japonicum and cor pulmonale. A pathologic study. Acta Med Philipp. 1965;2(2):68-77

46. King $\mathrm{CH}$, Dickman $\mathrm{K}$, Tisch DJ. Reassessment of the cost of chronic helmintic infection: a meta-analysis of disability-related outcomes in endemic schistosomiasis. Lancet. 2005:365(9470):1561-9.

47. Balen J, Zhao Z-Y, Williams GM, McManus DP, Raso G, Utzinger J, et al. Prevalence, intensity and associated morbidity of Schistosoma japonicum infection in the Dongting Lake region, China. Bull WHO. 2007;85(7):519-26.

48. Cooppan RM, Schutte CH, Mayet FG, Dingle CE, Van Deventer JM, Mosese PG. Morbidity from urinary schistosomiasis in relation to intensity of infection in the Natal Province of South Africa. Am J Trop Med Hyg. 1986;35(4):765-76.

49. Sukwa TY, Bulsara MK, Wurapa FK. The relationship between morbidity and intensity of Schistosoma mansoni infection in a rural Zambian community. Int J Epidemiol. 1986;15(2):248-51.

50. Ouma JH, Vennervald BJ, Butterworth AE. Morbidity in schistosomiasis: an update. Trends Parasitol. 2001;17(3):117-8.

51. Savioli L, Hatz C, Dixon H, Kisumku UM, Mott KE. Control of morbidity due to Schistosoma haematobium on Pemba Island: egg excretion and hematuria as indicators of infection. Am J Trop Med Hyg. 1990;43(3):289-95.

52. Andrade G, Bertsch DJ, Gazzinelli A, King CH. Decline in infection-related morbidities following drug-mediated reductions in the intensity of 
Schistosoma infection: A systematic review and meta-analysis. PLoS Neg Trop Dis. 2017;11(2):e0005372.

53. Chan MS, Guyatt HL, Bundy DAP, Medley GF. Dynamic models of schistosomiasis morbidity. Am J Trop Med Hyg. 1996;55(1):52-62.

54. Chan MS, Anderson RM, Medley GF, Bundy DA. Dynamic aspects of morbidity and acquired immunity in schistosomiasis control. Acta Trop. 1996;62(2):105-17.

55. Medley GF, Bundy DA. Dynamic modeling of epidemiologic patterns of schistosomiasis morbidity. Am J Trop Med Hyg. 1996;55(5 Suppl):149-58.

56. Chan MS, Bundy DA. Modelling the dynamic effects of community chemotherapy on patterns of morbidity due to Schistosoma mansoni. Trans R Soc Trop Med Hyg. 1997;91 (2):216-20.

57. Guyatt H. Different approaches to modelling the cost-effectiveness of schistosomiasis control. Mem Inst Oswaldo Cruz. 1998;93(Suppl 1):75-84.

58. King CH. It's time to dispel the myth of 'asymptomatic' schistosomiasis. PLoS Negl Trop Dis. 2015;9(2):e0003504.

59. Shane HL, Verani JR, Abudho B, Montgomery SP, Blackstock AJ, Mwinzi PNM, et al. Evaluation of urine CCA assays for detection of Schistosoma mansoni infection in western Kenya. PLoS Negl Trop Dis. 2011;5(1):e951.

60. King CH, Olbrych SK, Soon M, Singer ME, Carter J, Colley DG. Utility of repeated praziquantel dosing in the treatment of schistosomiasis in high-risk communities in Africa: A systematic review. PLoS Negl Trop Dis. 2011;5(9):e1321.

61. King CH. Parasites and poverty: The case of schistosomiasis. Acta Trop. 2010;113(2):95-104.

62. King $\mathrm{CH}$, Sturrock RF, Kariuki HC, Hamburger J. Transmission control for schistosomiasis - why it matters now. Trends Parasitol. 2006;22(12):575-82.

63. Pediatric praziquantel consortium. http://www.pediatricpraziquantel consortium.org/. Accessed 20 Feb 2017

64. Fulford AJ, Butterworth AE, Ouma JH, Sturrock RF. A statistical approach to schistosome population dynamics and estimation of the life-span of Schistosoma mansoni in man. Parasitology. 1995;110(Pt 3):307-16.

65. Mitchell KM, Mutapi F, Savill NJ, Woolhouse MEJ. Protective immunity to Schistosoma haematobium infection is primarily an anti-fecundity response stimulated by the death of adult worms. Proc Natl Acad Sci U S A. 2012; 109(33):13347-52.

66. Beltran S, Boissier J. Are schistosomes socially and genetically monogamous? Parasitol Res. 2009;104(2):481-3.

67. Beltran S, Boissier J. Schistosome monogamy: who, how, and why? Trends Parasitol. 2008;24(9):386-91.

68. May RM. Togetherness among schistosomes: its effects on the dynamics of the infection. Math Biosci. 1977;35(3):301-43.

69. Evans DS, King JD, Eigege A, Umaru J, Adamani W, Alphonsus K, et al. Assessing the WHO 50\% prevalence threshold in school-aged children as indication for treatment of urogenital schistosomiasis in adults in central Nigeria. Am J Trop Med Hyg. 2013;88(3):441-5.

70. Turner HC, Bettis AA, Dunn JC, Whitton JM, Hollingsworth TD, Fleming FM, Anderson RM. Economic considerations for moving beyond the Kato-Katz technique for diagnosing intestinal parasites as we move towards elimination. Trends Parasitol. 2017 In press

71. London declaration on neglected tropical diseases. http://unitingtocombatntds. org/resource/london-declaration. Accessed 20 Feb 2017.

72. World Health Organization, More than 500 million praziquantel tablets needed each year to treat schistosomiasis. http://www.who.int/neglected_ diseases/integrated_media/integrated_media_schistosomiasis_2011/en/. Accessed 20 Feb 2017.

73. Lamberton PH, Crellen T, Cotton JA, Webster JP. Modelling the effects of mass drug administration on the molecular epidemiology of schistosomes. Adv Parasitol. 2015;87:293-327.

74. Turner HC, Truscott JE, Hollingsworth TD, Bettis AA, Brooker SJ, Anderson RM. Cost and cost-effectiveness of soil-transmitted helminth treatment programmes: systematic review and research needs. Parasit Vectors. 2015;8:355.

75. Turner HC, Truscott JE, Bettis AA, Shuford KV, Dunn JC, Hollingsworth TD, et al. An economic evaluation of expanding hookworm control strategies to target the whole community. Parasit Vectors. 2015;8:570.

76. Turner HC, Truscott JE, Fleming FM, Hollingsworth TD, Brooker SJ, Anderson RM. Cost-effectiveness of scaling up mass drug administration for the control of soil-transmitted helminths: a comparison of cost function and constant costs analyses. Lancet Infect Dis. 2016;16(7):838-46.

77. Huyse T, Webster BL, Geldof S, Stothard JR, Diaw OT, Polman K, Rollinson D. Bidirectional introgressive hybridization between a cattle and human schistosome species. PLoS Pathog. 2009;5(9):e1000571.
78. Webster BL, Diaw OT, Seye MM, Webster JP, Rollinson D. Introgressive hybridization of Schistosoma haematobium group species in Senegal: Species barrier break down between ruminant and human schistosomes. PLoS Negl Trop Dis. 2013;7(4):e2110.

79. Webster JP, Molyneux DH, Hotez PJ, Fenwick A. The contribution of mass drug administration to global health: past, present and future. Phil Trans Roy Soc B: Biol Sci. 2014;369(1645):20130434.

80. Macpherson CNL, Craig PS. Animal reservoirs of schistosomiasis. In: Macpherson CNL, Craig PS, editors. Parasitic helminths and zoonoses in Africa. Dordrecht: Springer Netherlands; 1991. p. 224-36.

81. Downs JA, Mquta C, Kaatano GM, Mitchell KB, Bang H, Simplice $H$, et al. Urogenital schistosomiasis in women of reproductive age in Tanzania's Lake Victoria region. Am J Trop Med Hyg. 2011;84(3):364-9.

82. Da'dara AA, Harn DA. Elimination of helminth infection restores HIV-1C vaccine-specific T cell responses independent of helminth-induced IL-10. Vaccine. 2010;28(5):1310-7.

83. Savardekar LS, Balaiah D, Mali BN. Association of Schistosoma haematobium and human papillomavirus in cervical cancer: a case report. Acta Cytol. 2010;54(2):205-8.

84. Kjetland EF, Leutscher PD, Ndhlovu PD. A review of female genital schistosomiasis. Trends Parasitol. 2012;28(2):58-65.

85. Schistosomiasis: progress report 2001-2011, strategic plan 2012-2020, http://apps.who.int/iris/handle/10665/78074. Accessed 20 Feb 2017.

86. King CH, Magak P, Salam EA, Ouma JH, Kariuki HC, Blanton RE. Measuring morbidity in schistosomiasis mansoni: relationship between image pattern, portal vein diameter and portal branch thickness in large-scale surveys using new WHO coding guidelines for ultrasound in schistosomiasis. Trop Med Int Health. 2003;8(2):109-17.

87. DeStigter KV, King CH, Keating CE, Ouma JH, Siongok TK, Mahmoud AA. Effect of targeted mass treatment on intensity of infection and morbidity in schistosomiasis mansoni: seven-year follow-up of a community in Machakos, Kenya. Trans Assoc Am Physicians. 1989;102:209-12.

88. Bradley DJ, McCullough FS. Egg output stability and the epidemiology of Schistosoma haematobium. II. An analysis of the epidemiology of endemic S. haematobium. Trans R Soc Trop Med Hyg. 1973;67(4):491-500.

\section{Submit your next manuscript to BioMed Central and we will help you at every step:}

- We accept pre-submission inquiries

- Our selector tool helps you to find the most relevant journal

- We provide round the clock customer support

- Convenient online submission

- Thorough peer review

- Inclusion in PubMed and all major indexing services

- Maximum visibility for your research

Submit your manuscript at www.biomedcentral.com/submit
( Biomed Central 